\title{
Flow Buildup in a Mixed Phase of Quark-Gluon Plasma Plus Hadrons
}

\author{
Judith M. Peters and Kevin L. Haglin \\ Department of Physics, Astronomy \& Engineering Science, \\ St. Cloud State University, 720 Fourth Avenue South, St. Cloud, MN 56301 USA
}

(Dated: November 10, 2018)

\begin{abstract}
Transport processes are considered for their abilities to generate flow in a mixed-phase system of hadrons plus quark-gluon plasma exhibiting temperature and density gradients. Diffusive mechanisms are found to be the leading sources. Even after overcoming viscous effects, conductive and diffusive forces generate flow velocities for pions in a model using kinetic theory to within fifteen percent of light speed. Since such flow is not observed, this could possibly rule out the mixed phase in the evolution of high energy heavy ion collisions.
\end{abstract}

PACS numbers: $25.75 . \mathrm{Ld}, 12.38 . \mathrm{Mh}$

The ultrarelativistic nuclear many-body problem is challenging to study in the laboratory because system sizes are limited to roughly stable nuclei and the excited states are populated for about as long as it takes light to traverse the system. And yet, there is a wealth of physics information to be gleaned, if only there were a way to extract it from the collision zones created in heavy ion collisions. Experimental programs at the $\mathrm{Su}-$ per Proton Synchrotron (SPS) and at the Relativistic Heavy Ion Collider (RHIC) are rapidly advancing owing to continually developing tools for this extraction [1]. Various signals of hadronic and subhadronic degrees of freedom have been proposed and are being widely used. For example, strangeness is expected to be enhanced and hidden charm to be suppressed at high energy density; spacetime correlations for produced particles provide devices for spatial and temporal measuring; photons and dileptons are the best temperature and spectral probes one has; and collective expansion as an observable could provide a dynamical measure of the equation of state. We study mechanisms which generate flow, and focus on the mixed phase. We hope to establish the extent to which observed flow signals and existence of a mixed phase with gradients are inconsistent with each other.

Flow is clearly visible in momentum spectra at RHIC to a level roughly one-half to two-thirds the speed of light depending on the species and the degree of centrality of the reaction [2]. Such explosive evolution in the nuclear systems could probe salient features of underlying quark and gluon degrees of freedom. The inevitable return to hadronic matter from highly excited quark matter might proceed through a mixed phase. If so, and if the system has even modest temperature and density gradients in the mixed phase, we show that conductive and diffusive processes give rise to an effective mechanism for generating significant flow. So significant that they turn out to be the leading sources; much stronger than hydrodynamic flow from pressure gradients. Even after overcoming viscous effects, flow velocities from conduction and diffusion in a simple model reach within fifteen percent of light speed. These forces would superimpose and add to hydrodynamic forces, which are responsible for significant flow by themselves 3, 4]. One could point to this as evidence ruling out the mixed phase with a temperature gradient in the evolution of such nuclear many-body systems.

We begin with the system in a state very near equilibrium at $T_{c} \simeq 170 \mathrm{MeV}$. Local kinetic equilibrium is assumed but globally we allow a temperature gradient $d T / d r \simeq-1 \mathrm{MeV} / \mathrm{fm}$. This is the simplest assumption, although other profiles could be envisaged. We note that a temperature gradient is not inconsistent with hydrodynamics since a local maximum temperature different by as much as $70 \mathrm{MeV}$ from the average temperature has been estimated [5]. By this stage in the evolution of the nuclear collisions, probably 5-10 fm/c after maximum overlap, the system is roughly spherical in shape with a radius of $d=10 \mathrm{fm}$. For definiteness, let us indicate temperatures are central temperatures, and we here assume zero skin thickness. Two quark flavors plus gluons and prehadrons (forming hadrons) are assumed to be in local thermal equilibrium. In practice, this corresponds to densities of 5.5, 0.30, and 0.08 per $\mathrm{fm}^{3}$ for massless partons, pions and protons, respectively. It has been recently pointed out that under these conditions thermophoresis is responsible for generating significant flow [6].

Description of irreversible phenomena in systems displaced somewhat from equilibrium are dominated by thermodynamic forces and flows. The flows tend to smooth out the inhomogeneities introduced by the forces. One such example is heat conduction which is responsible for a force per unit area on each hadron given by

$$
F / A=-\frac{1}{3} n\langle u\rangle \lambda \frac{d\langle E\rangle}{d T} \frac{d T}{d r},
$$

where $n$ is the number density of partons in the quarkgluon plasma, $\langle u\rangle$ is the mean parton velocity, $\lambda$ their mean free path and $\langle E\rangle$ is their average energy. The net thermophoretic force (from conduction) acting on the hadrons due to parton dynamics is then given by

$$
F=\pi R^{2}(F / A)
$$


where $R$ is the hadron radius. Using elementary methods, one can estimate from here the result of this radially outward force in terms of the asymptotic hadron velocity expected in a picture relevant for high-energy heavy-ion collisions. In [6] Thoma found the final radial velocity for pions to be $v_{f}=0.81$ and for protons $v_{f}=0.40$, although see 17] for a correction.

We go beyond the effects of conduction and begin to study other transport processes in the presence of the same temperature gradient. If there were a temperature gradient, then the default scenario is to support a density gradient as well. Particles will of course diffuse from regions of high density to regions of lower density. Parton diffusion, we argue, is therefore responsible for a force per unit area

$$
F / A=-\frac{1}{3}\langle u\rangle \lambda\langle p\rangle \frac{d n}{d T} \frac{d T}{d r},
$$

where this time the spatial variation in density drives the mechanism. The average momentum appears rather than energy trivially, since force is proportional to $\dot{p}[\mathbf{7}]$.

Finally, the last effect we include is viscosity. Viscous effects are notoriously difficult to include in hydrodynamics, but here we use elementary kinetic theory argumentation to write

$$
F / A=\frac{1}{10} n\langle u\rangle \lambda \frac{d\langle p\rangle}{d T} \frac{d T}{d r} .
$$

Viscosity effects, like diffusion, involve average momentum rather than energy, as was the case for conduction which involves energy transport. In the massless limit the distinction disappears. Notice also that viscosity in this approach is $3 / 10$ of the conductive force and that the direction of the viscous force opposes conduction and diffusion [8]. Instead of using the simplified approach taken here, one could compute collision brackets in the first Chapman-Enskog approximation for linearizing the full parton kinetic theory [9]. However, the elegant results could get lost in the details. We also note that viscosity of a quark-gluon plasma has been estimated previously using transport theory [10 as well as kinetic theory [11.

Now we explore two different pictures: 1) a system of massless partons plus hadrons and, 2) a system of partons with dynamically-generated masses plus hadrons, with $m_{q}=g T / \sqrt{6}$ for quarks and antiquarks and $m_{g}$ $=g T / 2\left(1+N_{f} / 6\right)$ for gluons, where $N_{f}$ is the number of flavors [12, 13]. The idea is to compare the preliminary and rough estimates of conduction by Thoma to the more quantitative formalism discussed here, to compare also with diffusion, and finally, to explore finite-mass effects arising from the medium.

It is particularly convenient to first consider massless partons since the expressions can then be reported very compactly. To set the notation and indicate explicitly the partonic states considered, let $g_{q}=g_{\bar{q}}=N_{s} \times N_{c} \times N_{f}$ $=2 \times 3 \times 2=12$ and $g_{g}=N_{s} \times N_{c}=2 \times 8=16$ for the

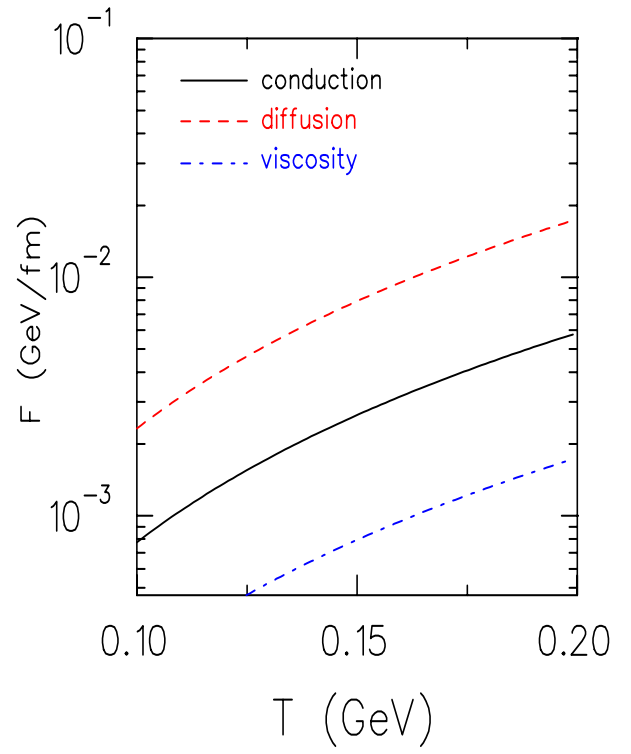

FIG. 1: Magnitudes of forces due to a temperature gradient. Heat conduction effects (solid curve), diffusion (dashed curve) and viscosity effects (dot-dashed curve) are shown separately.

degeneracies. Noting that $\lambda_{q}=\lambda_{\bar{q}}=4 / 9 \lambda_{g}$, we find

$$
\begin{aligned}
F / A)_{\mathrm{cond}} & =-\frac{T^{3}}{\pi^{2}} \zeta(4) \lambda_{q}\left[\frac{7}{4} g_{q}+\frac{4}{9} g_{g}\right] \frac{d T}{d r} \\
F / A)_{\mathrm{diff}} & =-\frac{T^{3}}{\pi^{2}} \zeta(4) \lambda_{q}\left[\frac{21}{4} g_{q}+\frac{4}{3} g_{g}\right] \frac{d T}{d r} \\
F / A)_{\mathrm{visc}} & =\frac{T^{3}}{\pi^{2}} \zeta(4) \lambda_{q}\left[\frac{21}{40} g_{q}+\frac{2}{15} g_{g}\right] \frac{d T}{d r}
\end{aligned}
$$

Since $\zeta(4)=\pi^{4} / 90$ 14], and since $\lambda_{q}=\left[3 C_{F} \alpha_{s} T\right]^{-1}$ (with the Casmir invariant $C_{F}=4 / 3$ ) has been computed in studies of parton relaxation rates using a hardthermal-loop (HTL) approximation [15], and finally plugging in the degeneracies, we arrive at

$$
\begin{aligned}
F / A)_{\mathrm{cond}} & =-\frac{\pi^{2} T^{2}}{4 \alpha_{s}}\left(\frac{253}{810}\right) \frac{d T}{d r}, \\
F / A)_{\mathrm{diff}} & =-\frac{\pi^{2} T^{2}}{4 \alpha_{s}}\left(\frac{253}{270}\right) \frac{d T}{d r} \\
F / A)_{\mathrm{visc}} & =\frac{\pi^{2} T^{2}}{4 \alpha_{s}}\left(\frac{253}{2700}\right) \frac{d T}{d r} .
\end{aligned}
$$

For simplicity, we assume spherical hadrons (prehadrons, or extended hadron bubbles) of radius $R \simeq 1 \mathrm{fm}$ and compute $F=\pi R^{2}(F / A)$ for the three transport mechanisms. The thermodynamic forces turn out to be proportional to the square of the temperature times the temperature gradient. However, we allow the strong coupling constant to run with temperature and approach an asymptotically free value in the $T \rightarrow \infty$ limit according to 16 ]

$$
\alpha_{s}(T)=\frac{\alpha_{s}\left(T_{c}\right)}{1+C \ln \left(T / T_{c}\right)},
$$




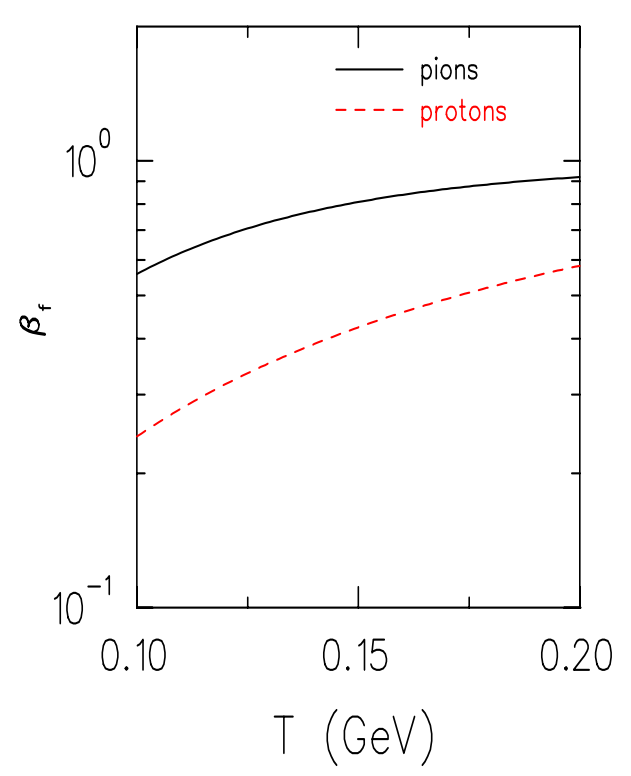

FIG. 2: Final flow velocities for pions (solid curve) and protons (dashed curve) generated by transport processes.

where $C \simeq 0.76, \alpha_{s}\left(T_{c}\right)=0.48$, and $T_{c}=170 \mathrm{MeV}$. The final dependence of the forces on temperature is softer than $T^{2}$ as shown in Fig. 1] The results suggest that diffusion seems to be responsible for the dominant force.

These radial forces act on prehadrons of mass $M$ and consequently generate radial flow. The force is constant so quite simply the final hadron kinetic energy is equal to the net force times the distance $d$, i.e. $K=$ $\left(F_{\text {cond }}+F_{\text {diff }}+F_{\text {visc }}\right) d\left(\right.$ since $\left.F_{\text {visc }}<0\right)$. Using relativistic kinematic formulæ, we find 17 .

$$
\beta_{f}=\sqrt{1-\left(\frac{M}{K+M}\right)^{2}} .
$$

The final flow velocities for pions and protons using $d=$ $10 \mathrm{fm}$ are plotted in Fig. 2] as a function of temperature. At $T=T_{c}=170 \mathrm{MeV}$ the values are 0.86 and 0.49 , respectively.

Next we explore the first-order effects of the medium to the extent that thermal masses could change these results. Relevant quantities appear in the form

$$
\langle\mathcal{O}\rangle=\int d^{3} p[\mathcal{O}] f\left(p_{0}\right) / \int d^{3} p f\left(p_{0}\right),
$$

where $p_{0}=\sqrt{\vec{p}^{2}+m^{2}}$ and where equilibrium distributions $f=1 /\left[\exp \left(\beta p_{0}\right) \pm 1\right]$ are taken as appropriate for Bosons (Fermions) using the $-(+)$ sign. We calculate

$$
\begin{aligned}
n & =\frac{g T^{3}}{2 \pi^{2}} \sum_{\ell=1}^{\infty} \frac{( \pm)^{\ell+1}}{\ell} x^{2} K_{2}(x \ell) \\
\langle p\rangle & =\frac{g T^{4}}{\pi^{2} n} \sum_{\ell=1}^{\infty} \frac{( \pm)^{\ell+1}}{\ell^{4}}\left[x^{2} \ell^{2}+3 x \ell+3\right]
\end{aligned}
$$

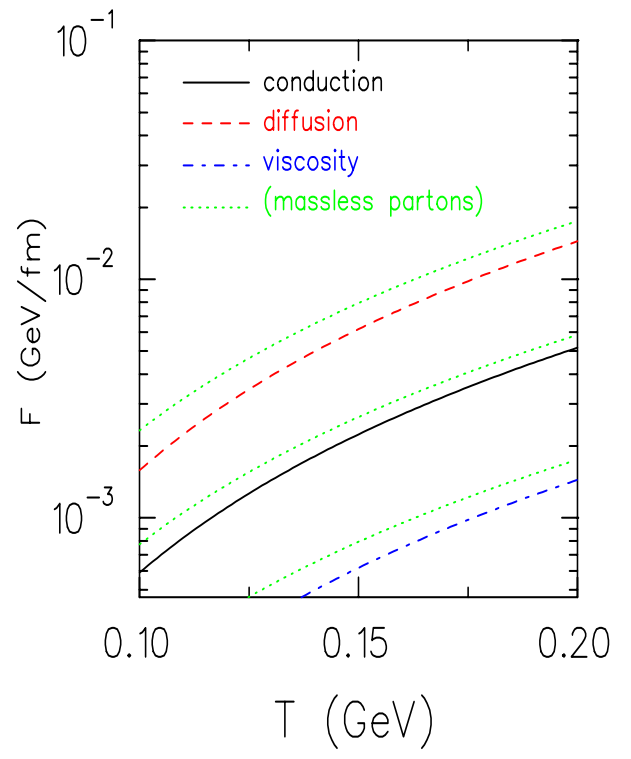

FIG. 3: Transport force magnitudes from massive quarks and gluons. Conduction is shown as the solid curve, diffusion is the dashed curve and viscosity is shown in the dot-dashed curve. For each curve, the massless parton limit is plotted as the dotted curve immediately above.

$$
\begin{aligned}
& \times e^{-x \ell} \\
\langle E\rangle= & \frac{g T^{4}}{2 \pi^{2} n} \sum_{\ell=1}^{\infty} \frac{( \pm)^{\ell+1}}{\ell^{2}}\left[x^{3} \ell K_{1}(x \ell)\right. \\
& \left.+3 x^{2} K_{2}(x \ell)\right] \\
\langle u\rangle= & \frac{g T^{3}}{\pi^{2} n} \sum_{\ell=1}^{\infty} \frac{( \pm)^{\ell+1}}{\ell^{3}}(x \ell+1) e^{-x \ell},
\end{aligned}
$$

where $x \equiv m \beta=m / T, K_{i}$ is the modified Bessel function of order $i, g$ is the appropriate degeneracy, $n$ is the density of quarks or gluons, $\langle u\rangle$ is the average speed and the choice of sign in the summands corresponds to + for gluons (Bosons) and - for quarks (Fermions). The massless limits for the above expressions are naturally recovered when $x \rightarrow 0$ since

$$
\begin{aligned}
\lim _{z \rightarrow 0}\left(z K_{1}(z)\right) & =1 \\
\lim _{z \rightarrow 0}\left(z^{2} K_{2}(z)\right) & =2 .
\end{aligned}
$$

In that case $\langle p\rangle=\langle E\rangle$ and $\langle u\rangle=1$. Truncation of the sums using only $\ell=1$ terms corresponds to the Boltzmann limit. Temperature derivatives of the quantities in Eq. (10) can be readily computed as needed.

The first observation regarding the thermal masses is that the effects on forces shown in Fig. 3 are quite small (at the ten percent level). This insensitivity to medium effects on the forces and the expected flow is consistent with previous findings 18. The effects are slightly more apparent at lower temperatures mainly due to the density. 


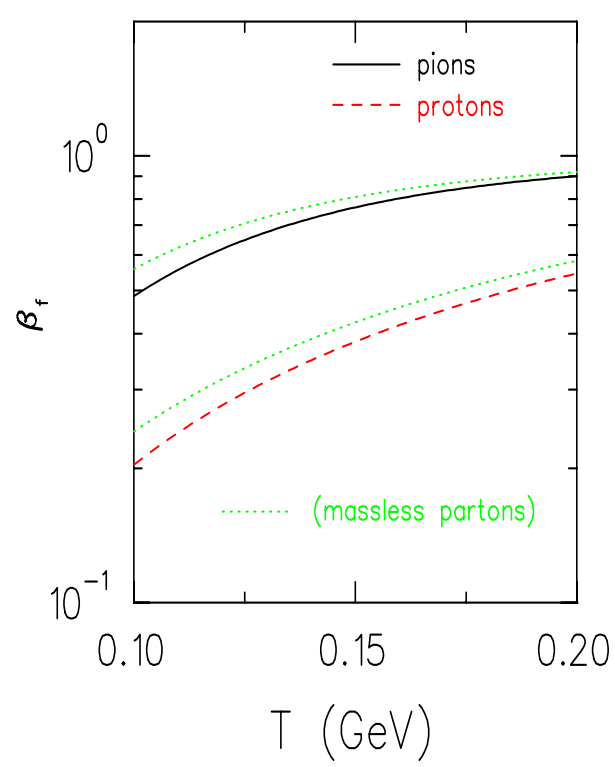

FIG. 4: Final flow velocities due to thermal-parton transport forces for pion (solid curve) and protons (dashed curve). Results for massless partons are also shown for each case (dotted curves).

For a symmetrical presentation of the results, we include Fig. 4 which reports the final flow velocities of hadrons (pions and protons) due to transport forces from massive partons. Results using massless partons obtained previously are shown as well in the figure.

Before concluding, a remark is in order regarding the choice of gradient and system size. Since the final hadron kinetic energy is equal to the product of the temperature gradient times the system size $d$, the same results would emerge if one instead took $d=1 \mathrm{fm}$, for then $d T / d r$ would be $-10 \mathrm{MeV} / \mathrm{fm}$. Consequently, the expected flow would be unchanged.

We summarize our study as follows. Transport processes have been studied in a mixed phase of quarks and gluons plus hadrons at or near $T_{c}$. A modest temperature gradient is responsible for a net radial force on the hadrons having magnitude $11 \mathrm{MeV} / \mathrm{fm}$. The leading mechanism or source of flow is parton diffusion which pushes hadrons radially outward roughly $9 \mathrm{MeV} / \mathrm{fm}$. Viscosity introduces a drag force of $\sim 1 \mathrm{MeV} / \mathrm{fm}$. In a simple picture, these forces are responsible for flow velocities $0.49 c$ for protons and $0.86 c$ for pions. The first-order effect of the medium was included, in which case the forces dropped by 10-20 percent. Flow velocities were consequently scaled back slightly as well, although relativistic velocity dependences on forces are clearly nonlinear.

One might suggest that these result argue for the nonexistence of the mixed phase, since flow velocities greater than $0.6 c$ are not seen in the data at RHIC. Instead, the expansion and evolution of the fireball in heavy ion collisions could proceed very quickly through the phase boundary and smoothly as in say, a second order (or higher) phase transition from quark-gluon plasma to hadronic matter.

Acknowledgment. We thank Markus H. Thoma for useful discussions. This work was supported in part by the National Science Foundation under grant number PHY-0098760.

[1] See, for example, Quark Matter 2002 proceedings and the experimental contributions therein, Nucl. Phys. A715, (2003).

[2] J. M. Burward-Hoy, for the PHENIX Collaboration, Nucl. Phys. A715, 498c (2003).

[3] E. V. Shuryak, Nucl. Phys. A638, 207c (1998).

[4] D. Teaney, J. Lauret and E. V. Shuryak, Phys. Rev. Lett. 86, 4783 (2001).

[5] P. Huovinen, P. Ruuskanen, and S. Rasanen, Phys. Lett. B535, 1090 (2002).

[6] M. H. Thoma, Phys. Rev. Lett. 88, 202303 (2002).

[7] F Reif, Fundamentals of Statistical and Thermal Physics, McGraw-Hill, Inc., 483 (1965).

[8] The coefficients out in front of Eqs. (11) and (4) are not rigorously identified here. The best one can really do without a proper calculation of the transport coefficients is to indicate that the prefactors are of order 1 .

[9] S. R. de Groot, W. A. van Leeuwen and Ch. G. van Weert, Relativistic Kinetic Theory: Principles and Applications, North Holland Publishing Co., 168 (1980).

[10] G. Baym, H. Monien, C. J. Pethick and D. G. Ravenhall, Phys. Rev. Lett. 64, 1867 (1990).

[11] M. H. Thoma, Phys. Lett. B269, 144 (1991).

[12] M. Le Bellac, Thermal Field Theory, Cambridge University Press, 146 (1996).

[13] J. Letesslier and J. Rafelski, Hadrons and Quark-Gluon Plasma, Cambridge University Press, 305 (2002).

[14] M. Abramowitz and I. A. Stegun, Handbook of Mathematical Functions, Dover Publications, Inc., NY, 807 (1972).

[15] M. H. Thoma, Phys. Rev. D 49, 451 (1993).

[16] J. Letesslier and J. Rafelski, Hadrons and Quark-Gluon Plasma, Cambridge University Press, 286 (2002).

[17] Comparing the development here to Eq. (3) in Ref. 6], we notice that an incorrect assumption was implemented there converting the derivative from a time to a spatial derivative. The erroneous assumption is a constant velocity. Therefore, Eqs. (4) and (5) of Ref. [6] are slightly incorrect and our Eq. (8) is the correct one.

[18] L. Csernai and R. Rohrich, Phys. Lett. B458, 454 (1999). 